ARTICLE

\title{
Cytokinin regulates vegetative phase change in Arabidopsis thaliana through the miR172/TOE1- TOE2 module
}

\author{
Sören Werner (iD) ${ }^{1}$, Isabel Bartrina (iD) ${ }^{1,2} \&$ Thomas Schmülling (iD) ${ }^{1 凶}$
}

During vegetative growth plants pass from a juvenile to an adult phase causing changes in shoot morphology. This vegetative phase change is primarily regulated by the opposite actions of two microRNAs, the inhibitory miR156 and the promoting miR172 as well as their respective target genes, constituting the age pathway. Here we show that the phytohormone cytokinin promotes the juvenile-to-adult phase transition through regulating components of the age pathway. Reduction of cytokinin signalling substantially delayed the transition to the adult stage. ZZ-type cytokinin was particularly important as compared to iP- and the inactive $c Z$-type cytokinin, and root-derived $t Z$ influenced the phase transition significantly. Genetic and transcriptional analyses indicated the requirement of SQUAMOSA PROMOTER BINDING PROTEIN-LIKE (SPL) transcription factors and miR172 for cytokinin activity. Two miR172 targets, TARGET OF EAT1 (TOE1) and TOE2 encoding transcriptional repressors were necessary and sufficient to mediate the influence of cytokinin on vegetative phase change. This cytokinin pathway regulating plant aging adds to the complexity of the regulatory network controlling the juvenile-to-adult phase transition and links cytokinin to miRNA action.

\footnotetext{
${ }^{1}$ Institute of Biology/Applied Genetics, Dahlem Centre of Plant Sciences (DCPS), Freie Universität Berlin, Albrecht-Thaer-Weg 6, 14195 Berlin, Germany.

${ }^{2}$ Present address: Institute of Biology, University of Graz, Schubertstrasse 51, 8010 Graz, Austria. ${ }_{\text {email: t.schmuelling@fu-berlin.de }}$
} 
$\mathrm{F}$ lowering plants progress through juvenile and adult phases of vegetative development before undergoing a transition to reproductive growth. The proper timing of these phases strongly influences plant fitness and reproductive success. Phase transitions are controlled by exogenous factors such as light (period and intensity) and temperature, as well as endogenous cues such as carbohydrate assimilates (mainly sucrose), hormones (e.g., gibberellin (GA)) and plant age feeding into intrinsic genetic programs ${ }^{1-3}$.

The transition from the juvenile to the adult vegetative phase is characterized by changes of shoot morphology as well as an increase in reproductive potential. In Arabidopsis thaliana, vegetative phase change is accompanied by an increase in size and length/width ratio of the leaf blade, an increase in the degree of serration of the leaf margins and a decrease in cell size ${ }^{4,5}$. In addition to these gradual changes, the appearance of trichomes on the abaxial side of leaves has been commonly used to define the transition from the juvenile to the adult phase ${ }^{6}$.

Two evolutionary highly conserved miRNAs, miR156 and miR172, and their respective target genes are at the center of the genetic mechanisms regulating the juvenile-to-adult phase transition ${ }^{7,8}$. The precursors of these miRNAs are encoded by ten (MIR156A-J) and five genes (MIR172A-E), respectively ${ }^{9,10}$. As the plant ages, the level of miR156 and the partly redundant miR157 decreases $^{11-13}$. They target ten out of 16 SQUAMOSA PROMOTER BINDING PROTEIN-LIKE (SPL) genes, a group of transcription factor genes controlling several aspects of Arabidopsis shoot development, such as the timing of juvenile-to-adult and vegetative-to-reproductive phase transitions, leaf initiation rate, and floral organ development ${ }^{14-16}$. In contrast to miR156, miR157 has only a minor effect on SPL expression and shoot morphology ${ }^{13}$. MiR156/miR157 decline with progressing plant development results in an increment of SPL mRNA abundance and translation ${ }^{14,17}$. Since at least five out of the ten miR156/ miR157-targeted SPL genes are directly involved in promoting the transcription of MIR172 genes ${ }^{14}$, the abundance of miR172 increases with plant age ${ }^{18}$. The targets of miR172 include the floral organ identity gene APETALA2 (AP2) and the AP2-like genes SCHLAFMÜTZE (SMZ), SCHNARCHZAPFEN (SNZ), TARGET OF EAT1 (TOE1), TOE2, and TOE3, which encode transcriptional regulators known to act as repressors of vegetative phase change and transition to flowering ${ }^{18-22}$. Corresponding to their opposite expression patterns, miR156 and AP2-like genes promote the juvenile phase, whereas miR172 and SPL genes promote the transition to the adult and the reproductive phase, as well as the respective accompanying heteroblastic features ${ }^{13,23}$.

In transgenic plants overexpressing miR172 abaxial trichomes are produced earlier, whereas abaxial trichome formation is delayed in miR172 knockout mutants with MIR172A and MIR172B playing dominant roles in the regulation of epidermal identity ${ }^{11,24}$. Leaf shape however is morphologically inconspicuous in plants with altered miR172 levels, indicating that in contrast to miR156/SPL, the miR172/AP2-like module affects only a subset of the leaf traits changing in the course of the transition ${ }^{11}$. Among the miR172 target genes, TOE1 and TOE2 have a very strong impact on juvenile leaf number: knocking out one of them already decreases the number of leaves without abaxial trichomes significantly, and simultaneous loss of both genes reduces the number of juvenile leaves even to less than half compared to the wild type ${ }^{11}$. Not much is known about the degree of individual influence of the other four target genes ( $A P 2$, $S M Z, S N Z$, and TOE3), but all six target genes of miR172 have to be knocked out in order to phenocopy a miR172 overexpressor ${ }^{25}$.

The phytohormone cytokinin (CK) plays a role in multiple processes that influence the growth and development of root and shoot organs. Amongst others, CK regulates cell proliferation and differentiation, the size and activity of apical meristems, photomorphogenesis, apical dominance, phyllotaxis, flowering time, and leaf senescence ${ }^{26}$. CK regulates also numerous processes related to nutritional cues and biotic and abiotic stress responses ${ }^{27}$.

CKs are $N^{6}$-substituted adenine derivatives whose metabolism and signal perception and transduction are largely known ${ }^{28}$. Isopentenyltransferases (IPTs) catalyze the formation of isopentenyladenine (iP) and cis-zeatin $(c \mathrm{Z})$ ribotides, the former of which can be hydroxylated by the cytochrome P450 monooxygenases CYP735A1 and CYP735A2 to form trans-zeatin $(t \mathrm{Z})$ ribotides $^{29-32}$. These precursors are converted into the corresponding bioactive free bases by the LONELY GUY (LOG) phosphoribohydrolases ${ }^{33,34}$. Reduction of the levels of active CKs is achieved through irreversible degradation by CK OXIDASE/ DEHYDROGENASES (CKXs) or conjugation to sugar moieties, most commonly glucose, rendering them inactive ${ }^{35,36}$. The $\mathrm{CK}$ signal transduction pathway is a multi-step His-Asp phosphorelay by a two-component signaling system ${ }^{28}$. In Arabidopsis, three membrane-bound histidine kinases serve as CK receptors: ARABIDOPSIS HISTIDINE KINASE2 (AHK2), AHK3, and CYTOKININ RESPONSE 1 (CRE1), also known as AHK4. CK binding to the receptor proteins results in an autophosphorylation of their kinase domains and the intramolecular transfer of the phosphoryl group to the receiver domain ${ }^{37-40}$. Histidine phosphotransfer proteins (AHPs) shuttle the phosphoryl group from the cytosol to the nucleus, where they activate type-B response regulators (ARRs). Acting as transcription factors, the type-B ARRs mediate the transcriptional output of the $\mathrm{CK}$ response. Among the primary response genes are type-A ARR genes encoding feedback inhibitors of the $\mathrm{CK}$ signaling pathway ${ }^{41,42}$.

Little is known about the influence of phytohormones on the juvenile-to-adult phase transition except for GA. GA was shown to promote the expression of some SPL genes and MIR172B and positively influence vegetative phase change ${ }^{6,43-47}$. Here, we describe a role for $\mathrm{CK}$ in the age-dependent regulation of vegetative phase change. We identified the most important components of the CK signaling pathway involved, as well as the convergence point with the age pathway. CK promotes the expression of miR172 genes, most probably involving SPL proteins. The miR172 targets TOE1 and TOE2 are necessary and sufficient to mediate the effect of CK on the juvenile-to-adult phase transition. Overall, this work describes the molecular basis for the influence of $\mathrm{CK}$ on vegetative phase change regulated by the age pathway.

\section{Results}

Cytokinin is a positive regulator of vegetative phase change. In Arabidopsis, the first rosette leaves are small, unserrated and round-shaped. With advancing age, leaf blade size and serration increase with every newly formed leaf ${ }^{23}$. Comparing the leaf morphology of mutants with a lower CK status like the CK receptor mutant ahk2 ahk3 and plants overexpressing the CKdegrading enzyme CKX1 (CKX1ox) with wild type, we observed a larger number of leaves with juvenile features (Fig. 1). In order to investigate the possible influence of $\mathrm{CK}$ on vegetative phase change, we examined the appearance of abaxial trichomes in CKX1ox and ahk2 ahk3, as well as in $c k x 3,4,5,6$ and the AHK2 gain-of-function mutant rock $2^{48}$ as plants with an increased CK content or signaling, respectively. In addition, we investigated a possible dependence of the phenotype on photoperiod since a long photoperiod stimulates abaxial trichome formation ${ }^{46}$. Plants were grown under both long-day (LD; $16 \mathrm{~h}$ of light, $8 \mathrm{~h}$ of darkness) and short-day conditions (SD; $8 \mathrm{~h}$ of light, $16 \mathrm{~h}$ of 

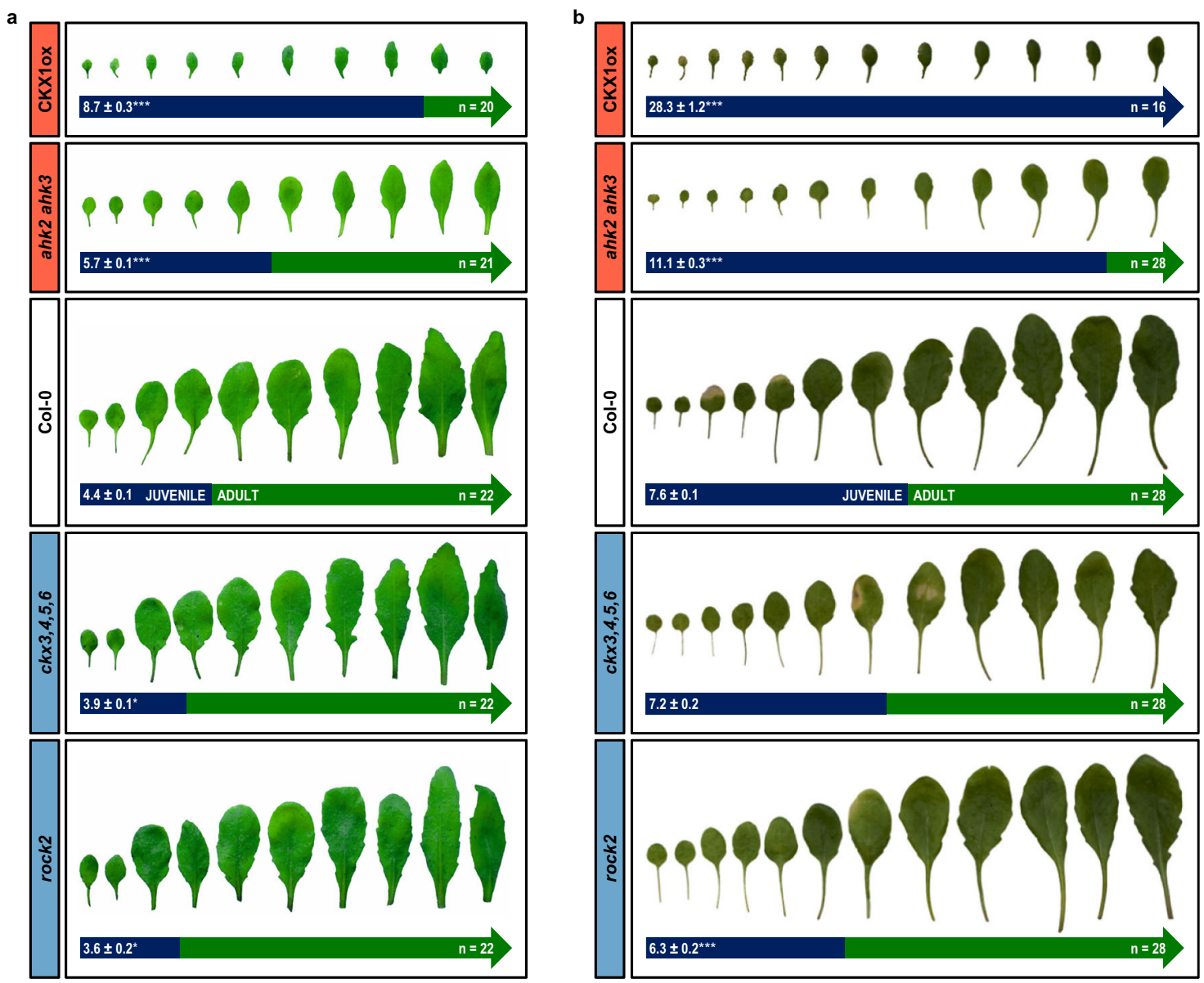

Fig. 1 Cytokinin promotes the juvenile-to-adult phase transition in a photoperiod-independent manner in Arabidopsis. a Morphology of the first ten leaves of Arabidopsis lines with an altered CK status grown in LD. b Morphology of the first 12 leaves of Arabidopsis lines with an altered CK status grown in SD. The color of the arrow indicates juvenile leaves (blue) and adult leaves (green). Values indicate the mean number of leaves without abaxial trichomes \pm SEM. Asterisks indicate significant differences compared to the wild type of the respective experiment, as calculated by Kruskal-Wallis test $\left({ }^{*} q<0.05\right.$; $\left.{ }^{\star \star} q<0.01 ;{ }^{\star \star \star} q<0.001\right)$. Exact $q$-values are included in the Source Data files.

darkness). We observed the same positive correlation of the altered CK status and the onset of the adult phase under both photoperiods, with more pronounced phenotypic differences under SD. Genotypes with a higher CK status displayed an earlier transition to the adult phase, whereas a reduced CK status prolonged the juvenile phase (Fig. 1).

Among the tested genotypes, the earliest transition to the adult phase was observed in the rock2 mutant: under SD conditions rock2 plants produced an average of $6.3 \pm 0.2$ juvenile leaves compared to $7.6 \pm 0.1$ juvenile leaves in wild type. Under LD conditions these were $3.6 \pm 0.2$ and $4.4 \pm 0.1$ juvenile leaves for these two genotypes. In contrast, ahk2 ahk3 plants formed $11.1 \pm 0.3$ and CKX1ox plants even $28.3 \pm 1.2$ leaves without abaxial trichomes under SD conditions. Ahk2 ahk3 and CKX1ox plants grown under LD conditions generated $5.7 \pm 0.1$ and $8.7 \pm 0.3$ juvenile leaves, respectively (Fig. 1 ).

Taken together, the results showed a positive influence of CK on the transition from the juvenile to the adult vegetative phase, which was photoperiod-independent.

CK genes involved in the regulation of vegetative phase change. In order to determine which CK genes of Arabidopsis might be functionally relevant, a series of mutants with altered CK metabolism or signaling causing a lower or higher CK status was analyzed for the appearance of abaxial trichomes under SD conditions. Among the mutations affecting CK biosynthesis and transport, a delay of the vegetative phase transition was only observed when the biosynthesis or the transport of $t \mathrm{Z}$-type CK was affected (ipt3,5,7; cypDM; abcg14) whereas plants lacking cZ-type CK (ipt2,9) behaved similar to wild type (Table 1). Mutations in the biosynthesis genes LOG4 and LOG7, which are expressed in the shoot apical meristem (SAM) and most likely provide the active CK for normal shoot meristem regulation ${ }^{49,50}$, did not alter juvenile leaf number (Table 1). Interestingly, plants lacking three $L O G$ genes $(\log 3,4,7)$ showed a tendency to a shorter juvenile phase (Table 1), contrasting the other results. Loss of four $C K X$ genes, including the SAM-expressed $C K X 3$ and $C K X 5^{51}$, resulted in a weak, but reproducible reduction in juvenile leaf number (Fig. 1 and Table 1), whereas the $c k x 3 c k x 5$ double mutant did not show any changes compared to wild-type plants (Table 1).

Among the three receptor double mutants only ahk2 ahk3 displayed a prolonged juvenile phase compared to the wild-type control, whereas ahk 2 cre 1 and ahk 3 cre 1 behaved like wild type (Fig. 2a). This suggests that AHK2 and AHK3 together control the CK-dependent juvenile-to-adult phase transition. Interestingly, among the mutants harboring a constitutively active cytokinin receptor, rock2 but not rock 3 produced a reduced number of juvenile leaves (Fig. 2a), indicating that AHK2 might be more relevant than AHK3 to mediate the vegetative phase transition.

There are 11 type-B ARR genes in Arabidopsis specifying the transcriptional CK response downstream of the receptors. To test 


\begin{tabular}{|c|c|c|}
\hline & No. of leaves w/o abaxial trichomes & $n$ \\
\hline \multicolumn{3}{|l|}{ Experiment 1} \\
\hline Col-0 & $11.2 \pm 0.3$ & 24 \\
\hline $\log 4$ & $11.4 \pm 0.4$ & 25 \\
\hline $\log 4 \log 7$ & $10.8 \pm 0.4$ & 25 \\
\hline ipt2 ipt9 & $11.8 \pm 0.4$ & 25 \\
\hline ipt3,5,7 & $12.9 \pm 0.3^{\star \star}$ & 24 \\
\hline abcg14 & $13.3 \pm 0.3^{\star \star \star}$ & 25 \\
\hline cypDM & $15.5 \pm 0.3^{\star \star \star}$ & 25 \\
\hline \multicolumn{3}{|l|}{ Experiment 2} \\
\hline Col-0 & $7.9 \pm 0.3$ & 22 \\
\hline $\log 3,4,7$ & $6.3 \pm 0.2^{\star}$ & 24 \\
\hline ipt3,5,7 & $9.0 \pm 0.2^{\star}$ & 24 \\
\hline abcg14 & $9.2 \pm 0.3^{\star}$ & 21 \\
\hline cypDM & $11.8 \pm 0.5^{\star \star \star}$ & 21 \\
\hline CKX1ox & $21.4 \pm 0.5^{\star \star \star}$ & 21 \\
\hline \multicolumn{3}{|l|}{ Experiment 3} \\
\hline Col-O & $8.0 \pm 0.3$ & 23 \\
\hline$c k \times 3 c k \times 5$ & $7.8 \pm 0.3$ & 23 \\
\hline$c k \times 3,4,5,6$ & $7.3 \pm 0.2$ & 23 \\
\hline \multicolumn{3}{|l|}{ Experiment 4} \\
\hline Col-O & $10.9 \pm 0.4$ & 25 \\
\hline $\operatorname{arr} 3,4,5,6,8,9$ & $10.5 \pm 0.2$ & 25 \\
\hline ahp2,3,5 & $9.7 \pm 0.3^{\star \star}$ & 26 \\
\hline \multicolumn{3}{|l|}{ Experiment 5} \\
\hline Col-O & $8.6 \pm 0.2$ & 28 \\
\hline $\log 3,4,7$ & $7.7 \pm 0.3^{\star}$ & 28 \\
\hline ahp2,3,5 & $7.9 \pm 0.1^{\star}$ & 32 \\
\hline \multicolumn{3}{|l|}{ Experiment 6} \\
\hline Col-O & $8.3 \pm 0.3$ & 24 \\
\hline $\operatorname{arr} 3,4,5,6,8,9$ & $7.8 \pm 0.3$ & 25 \\
\hline
\end{tabular}

Values indicate the mean number of leaves without abaxial trichomes $\pm \mathrm{SEM}$. Asterisks indicate significant differences compared to the wild type of the respective experiment, as calculated by Kruskal-Wallis test (experiments 1-5) or Mann-Whitney test (experiment 6$)^{*}\left({ }^{\star} q<0.05\right.$ $\left.{ }^{\star *} q<0.01 ;{ }^{* \star} q<0.001\right)$. Exact $p$-values and $q$-values are included in the Source Data files.

their involvement, we analyzed mutants of the ARR1, ARR2, $A R R 10, A R R 11$, and $A R R 12$ genes, as well as all their double and triple mutant combinations, since these type-B $A R R$ genes have the broadest expression profiles in the shoot ${ }^{52,53}$. Neither the single (Supplementary Fig. 1a) nor the double type-B $A R R$ mutants (Supplementary Fig. 1b) displayed any significant change in the number of juvenile leaves compared to wild-type plants. Among the triple mutants, only arr 1,10,12 produced a significantly larger number of juvenile leaves $(17.5 \pm 0.7$ compared to $9.8 \pm 0.2$ juvenile leaves in wild type) (Fig. 2b), while all other combinations showed a similar number of juvenile leaves as wild type (Supplementary Fig. 1d-f). This fits to the predominant function of $A R R 1, A R R 10$, and $A R R 12$ in regulating vegetative shoot growth ${ }^{54,55}$.

Further CK signaling mutants were tested: The histidine phosphotransfer protein mutant ahp2,3,5 and the type-A $A R R$ mutant arr3,4,5,6,8,9 did not show strong differences in juvenile leaf number (Table 1 ).

All in all, the CK receptors AHK2 and AHK3 as well as the type-B ARRs ARR1, ARR10, and ARR12 were identified as mediators of the $\mathrm{CK}$ activity in the juvenile-to-adult phase transition, whereas no specificity was found for other components of the CK signaling pathway, suggesting a high functional redundancy. Modifications of the CK status in the SAM $(\log 4,7$; $c k \times 3,5)$ did not cause any significant alterations of juvenile leaf number.
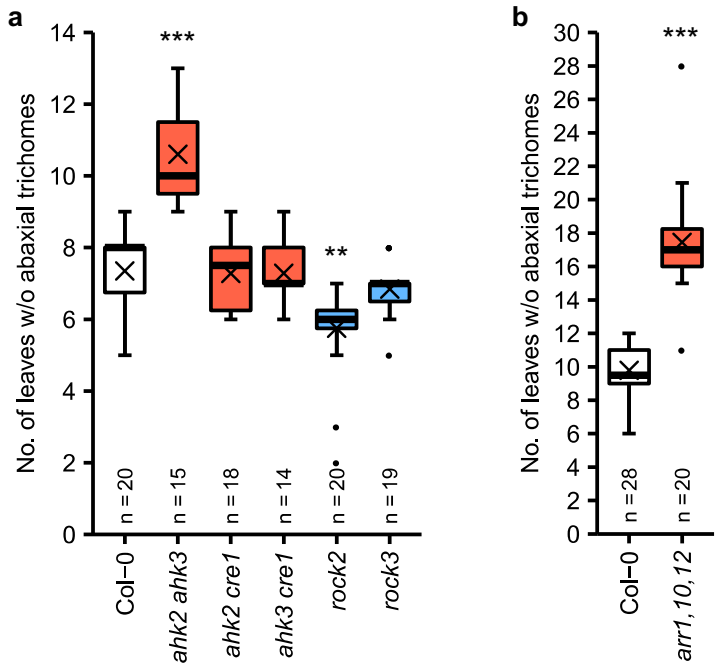

Fig. 2 The cytokinin-dependent regulation of the juvenile-to-adult phase transition is mediated by AHK2, AHK3, ARR1, ARR10, and ARR12. a, b Number of leaves without abaxial trichomes in cytokinin receptor mutants (a) and the type-B ARR mutant $\operatorname{arr} 1,10,12$ (b) grown in SD. In box plots, the center line represents the median value and the boundaries indicate the 25th percentile (upper) and the 75th percentile (lower). The $X$ marks the mean value. Whiskers extend to the largest and smallest value, excluding outliers which are shown as dots. Asterisks indicate significant differences compared to the wild type of the respective experiment, as calculated by Kruskal-Wallis test (a) or Mann-Whitney test (b) $\left({ }^{\star} p<0.05\right.$; ${ }^{\star \star} p<0.01$; $\left.{ }^{\star \star \star} p<0.001\right)$. Exact $p$-values are included in the Source Data files.

CK acts downstream of miR156. Vegetative phase change is regulated by a decrease in the abundance of the highly conserved master regulator miR156 ${ }^{11,12}$. In order to explore whether CK acts on vegetative phase change through miR156, we measured miR156 abundance 7, 14, and 21 days after germination in shoots of SDgrown plants with a higher or lower CK status. In agreement with previously published results, we observed an overall reduction of miR156 abundance with increasing age in the wild type (Fig. 3a and Supplementary Fig. 2a) ${ }^{11-13}$. The same pattern and expression level were also found in genotypes with a higher or lower CK status 7 and 14 days after germination. After 21 days however, CKX1ox showed a significantly elevated miR156 level compared to the wild type (Fig. 3a). Similar results were obtained for the primary transcripts of MIR156A and MIR156C (Supplementary Fig. 2b-e), which are the most important MIR156 genes for suppressing SPL activity during juvenile growth ${ }^{13}$, as well as for the partly redundant miRNA miR157 (Supplementary Fig. 2f, g).

To test a possible direct short-term influence of CK on miR156 abundance, we treated 10-day-old wild-type seedlings with $1 \mu \mathrm{M}$ 6-benzyladenine (BA) and measured the expression level at different time points after the treatment. The CK treatment caused a strong induction of several type-A ARR genes (Supplementary Fig. 3a), which are known CK response genes. In contrast, no significant changes in response to CK were noted neither for the abundance of miR156 (Fig. 3b) and miR157 (Supplementary Fig. 3b) nor for the transcript levels of MIR156A and MIR156C (Supplementary Fig. 3c).

Next, we crossed ahk2 ahk3 with the miR156 target mimicry line p35S:MIM156 (MIM156). The strong expression of an artificial miR156 target in this line reduces the inhibitory effect of miR156 on SPL expression resulting in the absence of a juvenile phase ${ }^{56,57}$. Intriguingly, the MIM156 transgene could not counterbalance the late appearance of abaxial trichomes in ahk2 ahk3 (Fig. 3c). To exclude that this is due to transgene silencing caused by the presence of several T-DNAs in the hybrid line we 
a

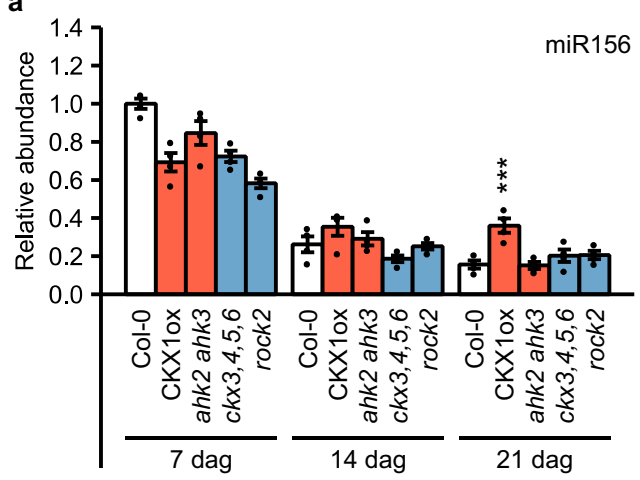

b

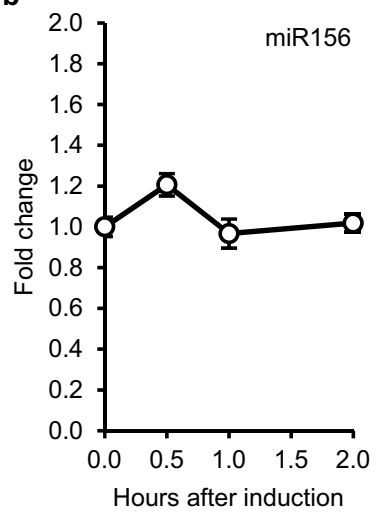

C

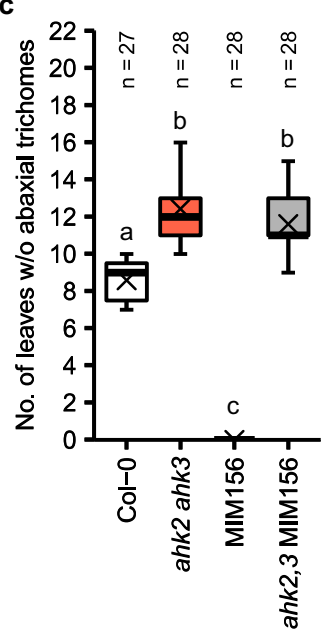

Fig. 3 The effect of cytokinin on the juvenile-to-adult phase transition is not dependent on miR156. a miR156 abundance in whole shoots of genotypes with an altered CK status compared to the wild type $(n=4$ biological replicates). Dots indicate each single biological replicate. Asterisks indicate significant differences compared to the wild type of the respective time point, as calculated by one-way ANOVA, post-hoc Dunnett's test $\left({ }^{\star} p<0.05 ;{ }^{\star \star} p<0.01 ;{ }^{\star \star \star} p<0.001\right)$. See also Supplementary Fig. 2a. b miR156 expression kinetics in 10-days-old SD-grown wild-type seedlings after treatment with $1 \mu \mathrm{M} \mathrm{BA}$ ( $n=6$ biological replicates). Statistical analysis was performed using one-way ANOVA, post-hoc Dunnett's test. No statistically significant differences were observed for miR156, comparing each time point with time point $0(p<0.05)$. Transcript levels $(\mathbf{a}, \mathbf{b})$ were determined by qRT-PCR. Data were normalized to TAFII15. Data displayed are expressed as mean \pm SEM. c Number of leaves without abaxial trichomes in ahk2 ahk3 MIM156 hybrid plants grown in SD. In the box plot, the center line represents the median value and the bounderies indicate the 25th percentile (upper) and the 75th percentile (lower). The $\mathrm{X}$ marks the mean value. Whiskers extend to the largest and smallest value, excluding outliers which are shown as dots. Letters indicate significant differences between the genotypes, as calculated by Kruskal-Wallis test $(p<0.05)$. Exact $p$-values calculated for $\mathbf{a}-\mathbf{c}$ are included in the Source Data files.

determined and confirmed MIM156 expression in ahk2,3 MIM156 (Supplementary Fig. 4). Consistently, the transcript levels of SPL genes were also increased in ahk2,3 MIM156 (Supplementary Fig. 5).

These results show that $\mathrm{CK}$ does not mediate its effect on vegetative phase change through miR156. In addition, the genetic analysis suggests that $\mathrm{CK}$ signaling is required to realize the action of miR156 on vegetative phase change, and that CK acts downstream of miR156.

SPLs are involved in the CK-dependent regulation of the juvenile-to-adult phase transition. The steady state mRNA levels of SPL genes that have been shown to play a major role in vegetative phase change ${ }^{14}$ (SPL2, SPL9, SPL10, SPL11, SPL13, and SPL15) were similar in ahk2 ahk3 and wild type (Supplementary Fig. 5). In order to analyze a possible immediate influence of CK on SPL gene expression, we measured their transcript levels in response to $\mathrm{CK}$. Because a potential $\mathrm{CK}$ effect on SPL gene expression could be masked eventually in wild type by the counteraction of miR156, we treated the MIM156 line with BA. Type-A ARR expression in MIM156 in response to CK was determined as an induction control, which showed the expected increase (Supplementary Fig. 6). In contrast, no significant changes of SPL transcript levels were detected after CK treatment (Fig. 4a).

To investigate further the potential role of SPLs in CKregulated phase transition, we carried out a genetic analysis and introgressed the early-transitioning rock2 mutation into the wellcharacterized spl9 spl15 mutant ${ }^{58}$. The rock 2 mutation did not rescue the delayed vegetative phase change of spl9 spl15 (Fig. 4b), suggesting that SPL9 and SPL15 are not or not the only SPL genes mediating the CK effect. Supporting this conclusion, the rock2 mutation reduced the percentage change of juvenile leaf number by a similar portion in both backgrounds (about $18 \%$ in wild type and $16 \%$ in spl9 spl15). To extend the analysis on all miR156targeted $S P L$ genes, we introgressed the rock 2 mutation into a transgenic line expressing p35S:MIR156B (MIR156ox), which allows no accumulation of SPL transcripts resulting in a substantially prolonged juvenile phase ${ }^{11,17,23,59}$. Rock2 was not able to accelerate the transition to the adult phase caused by p35S:MIR156B expression (Fig. 4c), indicating that regulation of vegetative phase change by CK relies on SPL function.

The influence of $\mathrm{CK}$ on vegetative phase change depends on miR172 as well as TOE1 and TOE2. SPLs are positive regulators of miR172 expression and consequently, the age-dependent increase of SPL levels causes a progressive elevation of miR172 abundance in wild-type plants ${ }^{11,12,18,21}$ (Supplementary Fig. 7a). An increase of miR172 with advancing age was also visible in plants with an altered CK status (Fig. 5a). However, it progressed slower in CK-deficient plants and faster in plants with a higher CK status. Small differences in miR172 abundance were already detectable 7 days after germination and became more prominent at later time points. Twenty-one days after germination only about half of the miR172 level of wild type was present in plants with a lower CK status. In contrast, plants with a higher CK status showed a higher miR172 level (Fig. 5a). Among the tested miR172 precursors, MIR172A and MIR172B, which were shown to play dominant roles in the timing of trichome initiation ${ }^{24}$, showed a similar expression pattern (Supplementary Fig. 7b-e).

Next, we tested the response of MIR172 gene expression and mature miR172 levels to CK. BA treatment resulted in an up to two-fold increase of MIR172A and MIR172B transcript levels within 1-2 h (Fig. 5b). The response of MIR172 gene expression to $\mathrm{CK}$ was also reflected by transiently increased mature miR172 levels upon $\mathrm{CK}$ treatment (Fig. 5c). In order to test the dependence of the CK-mediated regulation of vegetative phase change on miR172, we crossed ahk2 ahk3 with a MIR172B overexpression line (MIR172ox). For unknown reasons, we were unable to generate a homozygous hybrid line and also direct transformation of the transgene into the ahk2 ahk3 receptor mutant did not yield any primary transformants. However, transforming p35S:MIR172B into the less severe ahk2-5 ahk3-7 mutant $^{60}$ yielded transformants that were directly used for phenotyping under SD conditions. Since control lines could not 

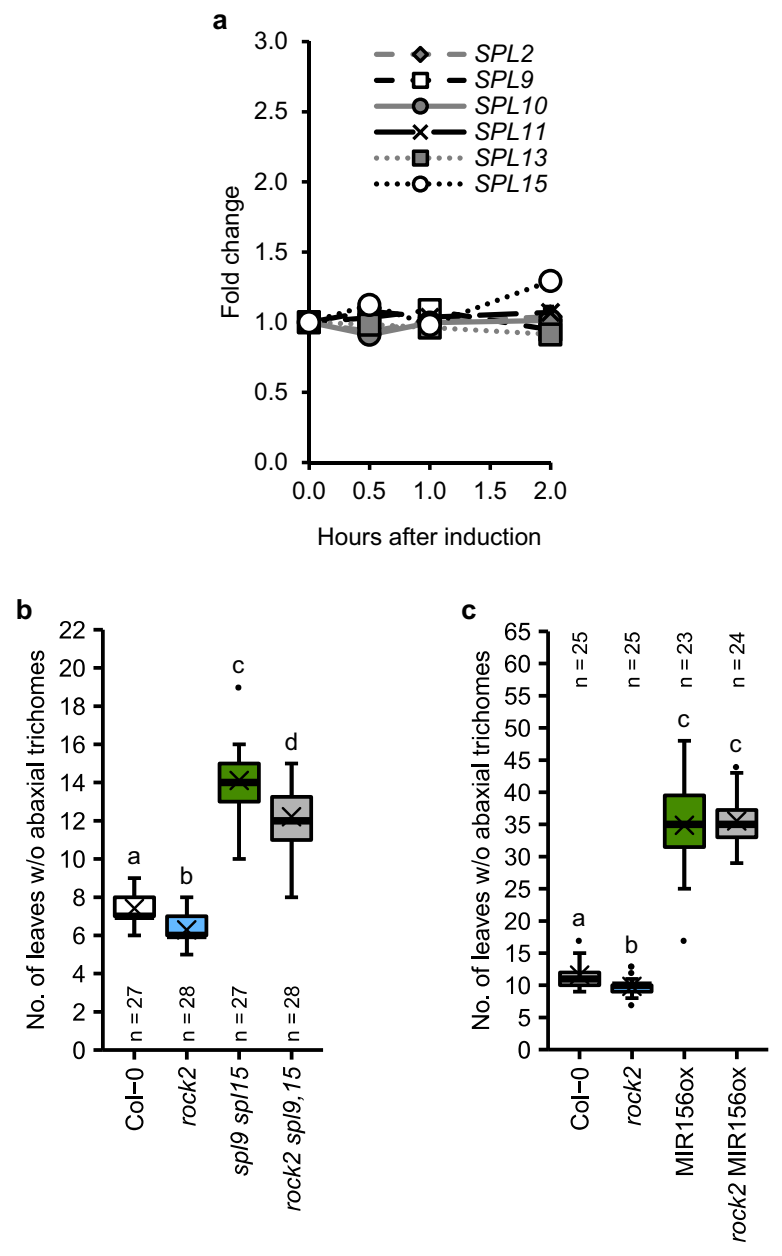

Fig. 4 SPLs are involved in the cytokinin-dependent regulation of vegetative phase change. a SPL expression kinetics in 10-day-old SDgrown MIM156 seedlings after treatment with $1 \mu \mathrm{M}$ BA. Transcript levels were determined by qRT-PCR. Data were normalized to TAFII15 and PP2AA2. Data displayed are expressed as mean \pm SEM $(n=6$ biological replicates). Statistical analyses were performed using one-way ANOVA, post-hoc Dunnett's test. No statistically significant differences were observed for any SPL gene, comparing each time point with time point 0 $(p<0.05)$. b, c Number of leaves without abaxial trichomes in rock2 spl9 spl15 plants (b) and rock2 MIR156ox plants (c) grown in SD. In box plots, the center line represents the median value and the boundaries indicate the 25th percentile (upper) and the 75th percentile (lower). The X marks the mean value. Whiskers extend to the largest and smallest value, excluding outliers which are shown as dots. Letters indicate significant differences between the genotypes, as calculated by Kruskal-Wallis test $(q<0.05)$. Exact $p$-values and $q$-values calculated for $\mathbf{a}$-c are included in the Source Data files.

be grown on kanamycin-containing media in this experiment, a control experiment was conducted separately, comparing Col-0 and ahk2-5 ahk3-7 with respective T1 plants harboring the empty vector, showing no impact of selective media on juvenile leaf number (Supplementary Fig. 8). Transgene expression and presence of mature miR172 was confirmed by qRT-PCR (Supplementary Fig. 9). Similar to ahk2-2tk ahk3-3 used for the other experiments, ahk2-5 ahk3-7 showed a reduction of MIR172B expression and miR172 abundance (Fig. 5a and Supplementary Figs. 7e and 9). Ahk2-5 ahk3-7 also displayed a late-transitioning phenotype which was completely suppressed by the $p 35 S: M I R 172 B$ transgene (Fig. $5 \mathrm{~d}$ ), supporting the hypothesis of CK influencing vegetative phase change by regulating miR172 expression.

The regulation of MIR172 expression by CK should have an impact on miR172 targets as well. Since the miR172-mediated silencing of AP2-like genes occurs mainly on the translational level ${ }^{18,19,61}$, we refrained from measuring transcript levels and conducted a genetic analysis instead. The mutation of SMZ did not alter juvenile leaf number, but reduced it in the $a h k 2 a h k 3$ background (Supplementary Fig. 10a), suggesting a role for SMZ in the CK-dependent regulation of vegetative phase change. Simultaneous loss of $S M Z$ and its close homolog $S N Z$ caused a slightly earlier transition to the adult phase in the wild type. Introgression of both mutant alleles into the ahk2 ahk3 background resulted also in an earlier juvenile-to-adult phase transition with the effect being larger than in wild type (Fig. 6a). This indicates that SMZ and SNZ are required for regulation of the juvenile-to-adult phase transition in plants with a lower CK status but that additional factors play a role.

Loss of single TOE1 or TOE2 gene functions in the background of $a h k 2 a h k 3$ also showed their participation in mediating the CK activity (Supplementary Fig. 10b). The juvenile leaf number of ahk2,3 toe 1,2 plants was indistinguishable from toe 1 toe 2 control plants (Fig. 6b). The additional disruption of the TOE3 gene in the $a h k 2 a h k 3$ background did not result in a further reduction of the number of juvenile leaves (Supplementary Fig. 10c). Furthermore, introgression of rock 2 into the toe 1 toe 2 mutant did not result in a further decrease in juvenile leaf number (Fig. 6c) showing a strong epistatic relationship. These findings strongly suggest that these two miR172 target genes take part in the CKdependent regulation of vegetative phase change.

\section{Discussion}

This work has revealed a pivotal role of $\mathrm{CK}$ in the control of vegetative phase change in Arabidopsis. Several key factors mediating the influence of $\mathrm{CK}$ on the juvenile-to-adult phase transition were identified and a link of the hormone to components of the age pathway is described (Fig. 7).

$\mathrm{IP}$ and $t \mathrm{Z}$ are generally considered to be the most active natural $\mathrm{CKs}$, whereas $c Z$ has mostly a lower activity ${ }^{62}$. Analysis of $\mathrm{CK}$ biosynthesis and transport mutants showed the importance of $t Z$-type $C K s$ in the regulation of vegetative phase change. The abcg14 mutant, which is impaired in the long-distance allocation of $t Z$-type CKs from the root to the shoot ${ }^{63,64}$, as well as the $t \mathrm{Z}$ biosynthesis mutant cypDM ${ }^{65}$ produced $\sim 15 \%$ and $\sim 30 \%$ more juvenile leaves than the wild type, respectively (Table 1). Both mutants compensate the lack of $t \mathrm{Z}$ at least in part by increased production of iP-type $\mathrm{CKs}^{63-65}$, but this was not sufficient to allow proper timing of vegetative phase change, suggesting a less important role for iP in this process. Moreover, the vegetative phase change in the ipt2,9 mutant was similar to wild type indicating that $c Z$-type CKs have no role in this process. Notably, the significant retardation of the transition to the adult phase in the abcg14 mutant established CK as a root-borne factor promoting vegetative phase change, which was shown previously to be initiated mainly in the SAM as well as in the leaves themselves ${ }^{66,67}$. However, compared to its role in promoting the transition to flowering under $\mathrm{SD}^{68,69}$ the root-derived portion of $t \mathrm{Z}$ appears to have a more limited role in regulating vegetative phase change.

The more important function of $t Z$-type CKs in the juvenileto-adult phase transition compared to iP-type CKs is in agreement with the CK affinities of the involved receptors AHK2 and AHK3. AHK3 has been shown to display a higher affinity to $t \mathrm{Z}$ than to $\mathrm{iP}^{70}$ and the in planta $\mathrm{CK}$ response mediated by $\mathrm{AHK} 2$ and $\mathrm{AHK} 3$ is triggered more strongly by $t \mathrm{Z}$ than by $\mathrm{iP}^{71}$. 
a

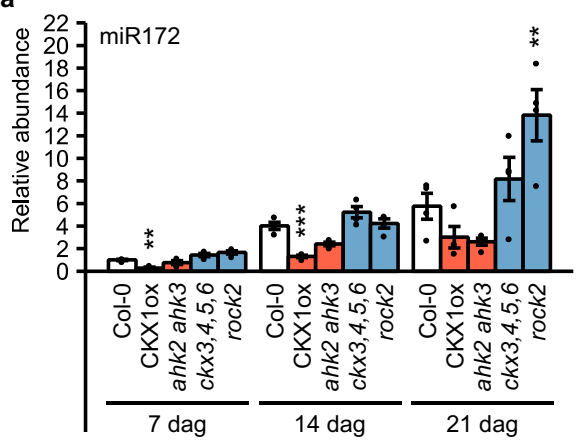

b

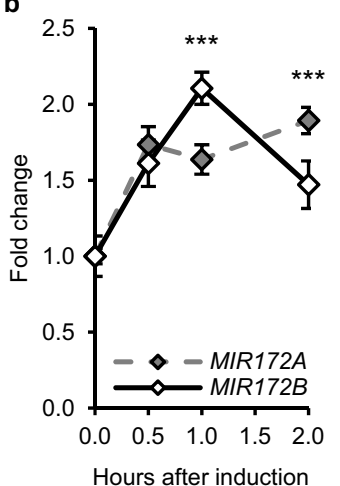

c

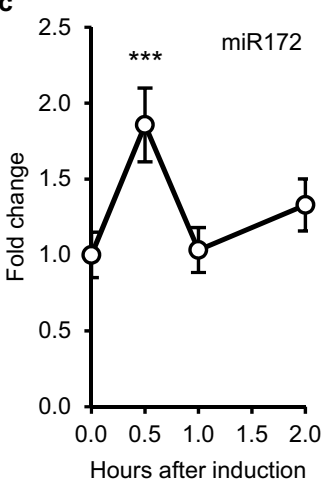

d

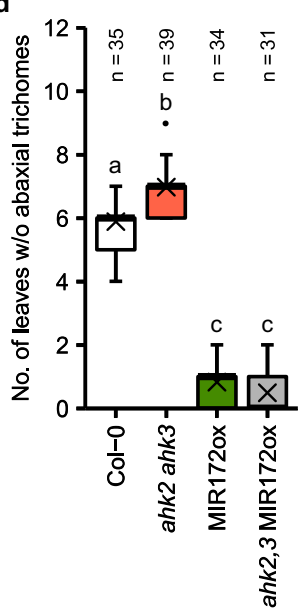

Fig. 5 Cytokinin increases miR172 abundance. a miR172 abundance in whole shoots of genotypes with an altered CK status compared to the wild type ( $n=4$ biological replicates). See also Supplementary Fig. 7a. Dots indicate each single biological replicate. b, c miR172 and MIR172 gene expression kinetics in 10-day-old SD-grown wild-type seedlings after treatment with $1 \mu \mathrm{M} \mathrm{BA}(n=6$ biological replicates). Transcript levels were determined by qRT-PCR. Data were normalized to TAFII15 (a, c) or TAFII15 and PP2AA2 (b). Data displayed are expressed as mean \pm SEM. Asterisks indicate significant differences compared to the wild type of the respective time point (a) or compared to time point $0(\mathbf{b}, \mathbf{c})$, as calculated by one-way ANOVA, post-hoc Dunnett's test $\left({ }^{\star} p<0.05 ;{ }^{* \star} p<0.01 ;{ }^{* \star *} p<0.001\right)$. d Number of leaves without abaxial trichomes in ahk2-5 ahk3-7 MIR172ox hybrid plants grown in SD. T1 plants shown in Supplementary Fig. 9 were used for the analysis. In box plots, the center line represents the median value and the boundaries indicate the 25th percentile (upper) and the 75th percentile (lower). The $X$ marks the mean value. Whiskers extend to the largest and smallest value, excluding outliers which are shown as dots. Letters indicate significant differences between the genotypes, as calculated by Kruskal-Wallis test $(q<0.05)$. Exact $p$-values and $q$-values calculated for a-d are included in the Source Data files.

Downstream of these receptors the type-B ARRs ARR1, ARR10, and ARR12 were identified to mediate the influence of CK on the juvenile-to-adult phase transition (Fig. 2b), which have been described previously as central regulators of shoot growth ${ }^{54,55,72}$. Notably, ARR2 has apparently no role (Supplementary Fig. 1d-f) despite its known functions in leaves ${ }^{73-75}$ where it mediates the inhibition of senescence by CK at a later developmental stage ${ }^{76}$. Taken together, the genetic analysis has shown the relevance of $t Z$-type CK for the juvenile-to-adult phase transition acting through the AHK2/AHK3-ARR1/ARR10/ARR12 signaling module.

Further analysis uncovered the participation of distinct components of the age pathway in mediating the action of CK. Transcriptional and genetic analysis argues against the involvement of miR156 in that process. MiR156 and also miR157 levels were not altered in CK mutants in early developmental stages or by exogenously applied CK (Fig. 3a, b and Supplementary Figs. $2 \mathrm{~g}$ and $3 \mathrm{~b}$ ). However, CK signaling was required to induce an early transition to the adult phase in the absence of the repressive master regulator miR156: Introgression of the late-transitioning ahk2 ahk3 double mutant into the MIM156 line completely abolished the effect of the target mimicry on the phenotype, while not substantially impacting the expression of the SPL transcript abundance in the hybrid line (Fig. $3 \mathrm{c}$ and Supplementary Fig. 5). It is evident, that $\mathrm{CK}$ does not affect vegetative phase change miR156-dependently, but acts downstream of it.

On the other hand, we showed that CK regulates the vegetative phase change miR172-dependently: MiR172 abundance positively correlated with the CK status in different $\mathrm{CK}$ mutants and increased transiently after CK treatment (Fig. 5a, c). Additionally, the ahk2 ahk3 mutant was unable to counteract the complete loss of the juvenile phase caused by expressing a $p 35 S: M I R 172 B$ transgene (Fig. 5d).

No changes in expression of miR156/miR157-regulated SPL genes were observed in the CK receptor mutant ahk2 ahk3 (Supplementary Fig. 5) or by treatment with exogenous CK (Fig. 4a). However, the inability of the rock2 mutation to counteract the effect of MIR156B overexpression (Fig. 4c) implies an involvement of SPLs in the CK-dependent regulation of vegetative phase change. Arabidopsis has 16 SPL genes, ten of which are targeted by miR156/miR15713,58,77,78. At least five of the SPLs induce MIR172 gene expression: SPL2, SPL9, SPL11, SPL13A/B, and SPL15 ${ }^{11,14}$. CK has a positive effect on miR172 abundance as well (Fig. $5 a-c$ and Supplementary Fig. $7 c$, e). These activities could be independent events, but it is also possible that SPLs and type-B ARRs or other CK signaling components induce MIR172 expression in a cooperative fashion. Both SPLs and typeB ARRs bind to MIR172 gene loci ${ }^{11,79-81}$. Furthermore, Zhang et al. ${ }^{82}$ showed physical interaction of SPL9 with several type-B ARRs, including ARR1, ARR10, and ARR12, causing a reduction of the CK-dependent shoot regeneration capacity. This repression of CK activity by SPLs contrasts with their aligned activities in promoting the juvenile-to-adult phase transition. But it could be that the consequences of the SPL-ARR interaction are contextspecific. The molecular mechanism of CK action through SPLs remains to be shown.

Yant et al. ${ }^{25}$ showed that all six miR172 target genes have to be mutated in order to mimic a miR172-overexpressing plant. Among the target genes, TOE1 and TOE2 have the strongest influence on the length of the juvenile phase, since mutating them reduces juvenile leaf number by more than half ${ }^{11}$ (Fig. 6b, c). Not much is known about the degree of influence of the other four genes. Loss of $S M Z$ and $S N Z$ only slightly reduced the number of juvenile leaves (Fig. 6a and Supplementary Fig. 10a) and AP2 and TOE3 are mostly known for their role in flower development ${ }^{21,83}$. The knockout mutants toe1, toe2, $s m z$, and $s m z$ snz all showed partial restoration of the juvenile phase of ahk2 ahk3 with toe 2 having the strongest impact (Fig. 6a and Supplementary Fig. 10a, b), whereas loss of both TOE1 and TOE2 completely suppressed the late transition of ahk2 ahk3 (Fig. 6b), indicating that TOE1 and TOE2 are both necessary and sufficient for the CK-regulation of the juvenile-to-adult phase transition.

Taken together, we added a regulatory layer to the agedependent control of vegetative phase change, with CK 


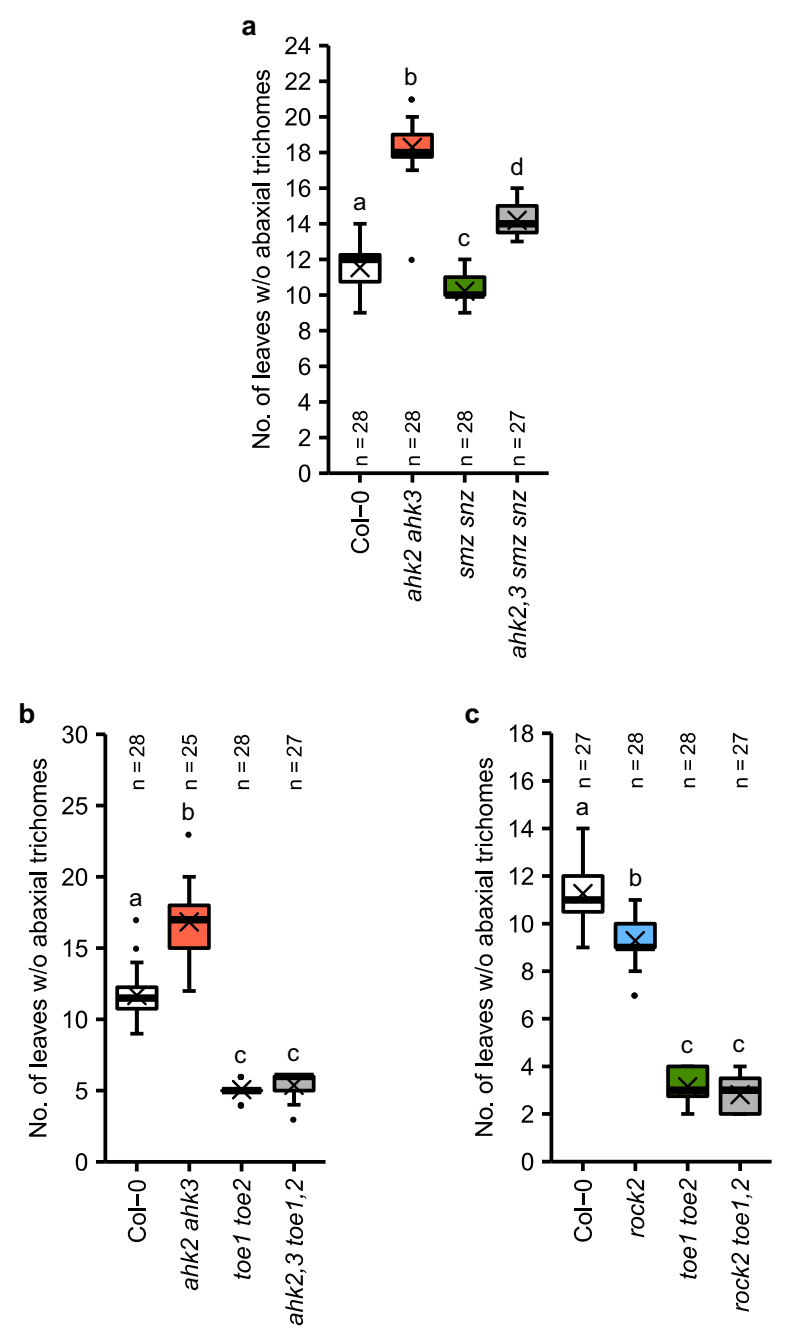

Fig. 6 Genetic interactions between AP2-like genes and cytokinin receptor genes regarding vegetative phase change. Number of leaves without abaxial trichomes in ahk2,3 smz snz (a), ahk2,3 toe 1,2 (b) and rock2 toe1,2 (c) hybrid plants grown in SD. In box plots, the center line represents the median value and the boundaries indicate the 25th percentile (upper) and the 75th percentile (lower). The $X$ marks the mean value. Whiskers extend to the largest and smallest value, excluding outliers which are shown as dots. Letters indicate significant differences between the genotypes, as calculated by Kruskal-Wallis test $(q<0.05)$. Exact $q$-values are included in the Source Data files.

promoting MIR172 gene expression (most probably in cooperation with SPLs), while not affecting the other key regulator miR156. Additionally, we identified TOE1 and TOE2 as being specifically important in the CK-dependent regulation of this process (Fig. 7).

Fouracre and Poethig 67 proposed that miR156 or a miR156derived signal produced by the SAM ensures juvenility of the first leaves, but as the plant grows, the identity of subsequent leaves is determined by peripheral organs to an increasing degree. Since CK acts on miR172 rather than miR156, CK would only affect later formed leaves, supporting the hypothesis that $\mathrm{CK}$ regulates vegetative phase change together with SPLs, whose expression is increased in later formed leaves when miR156 abundance decreases. Hence, CK might not be able to influence the identity of the first leaves, which explains why none of the tested mutants with a higher CK status show a reduction of juvenile leaf number as strong as toe 1 toe 2 or even p35S:MIR172 or p35S:MIM156

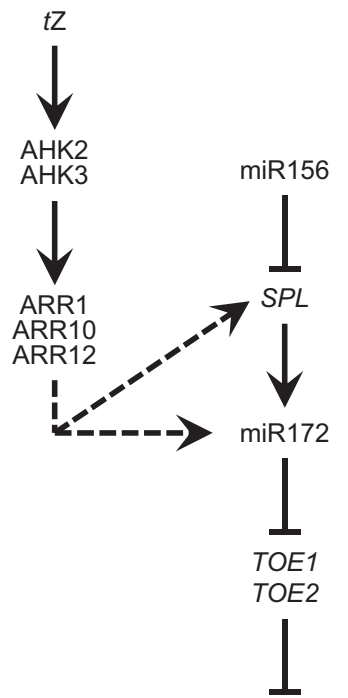

vegetative phase change

Fig. 7 Model of the cytokinin-dependent regulation of vegetative phase change. The $C K$ receptors $A H K 2$ and $A H K 3$ as well as the type- $B$ response regulators ARR1, ARR10, and ARR12 are activated mainly by $t Z$. Activation of CK signaling in the shoot results in an increase of miR172 abundance. MiRNA activation may be achieved directly by type-B ARRs or involve coaction of SPLs. TOE1 and TOE2 are repressed posttranscriptionally upon miR172 increment, enabling the transition from the juvenile to the adult vegetative phase.

plants. This hypothesis is supported by the fact that all CK signaling components identified in this study to be involved in regulating vegetative phase change are highly expressed in leaves $^{52,53,84}$. Furthermore, the importance of CK activity in the SAM for vegetative phase change is low, since loss of CK genes known to be active in the SAM (CKX3 and CKX $5^{51}$; LOG4 and $L O G 7^{50}$ ) did not cause any alterations in juvenile leaf number (Table 1).

Future work should explore the functional relevance of CK under different environmental conditions. A predominant activity of CK in regulating the juvenile-to-adult transition in later formed leaves would be consistent with the idea that the hormone mediates responses to environmental cues ${ }^{27}$, which are partly known to regulate the juvenile-to-adult phase transition ${ }^{85-87}$ to adapt to environmental changes ${ }^{88}$. Additionally, the question should be addressed whether other leaf traits characterizing vegetative phase change ${ }^{6}$ respond with similar sensitivity to an altered CK status as does epidermal identity analyzed here. MiR156 inhibits the development of all adult traits, with different SPLs promoting different subsets of these traits ${ }^{11}$, while TOE1 and TOE2 are only involved in the formation of abaxial trichomes ${ }^{11,25,89}$. Furthermore, the nature of the ARR-SPL interaction remains to be clarified, which might uncover other age-related processes regulated by the interplay of the two pathways.

\section{Methods}

Plant material and growth conditions. The Columbia- 0 (Col- 0 ) ecotype of Arabidopsis thaliana was used as the wild type. All mutants and transgenic lines that were used in this study and generated by crossings are listed in Supplementary Table 1. As a $s m z$ mutant, we used a previously uncharacterized knock-out allele (SALK_135824C) which was named $s m z-4$. No full-length $S M Z$ transcript was detected in this mutant by semi-quantitative RT-PCR, suggesting that this is a null allele (Supplementary Fig. 11). Primers used for the analysis of $s m z-4$ are listed in Supplementary Table 2. The mutant alleles used for the generation of the $c k x 3,4,5,6$ quadruple mutant were described in Bartrina et al. ${ }^{51}$. All genotypes were 
propagated under LD conditions ( $16 \mathrm{~h}$ dark $/ 8 \mathrm{~h}$ light cycle), $22^{\circ} \mathrm{C}$ and $30-65 \%$ humidity, and confirmed by PCR analysis. Primers used for genotyping are listed in Supplementary Table 3. For the analyses of juvenile leaf number and the quantification of miRNA abundance and gene expression in CK mutants, Arabidopsis plants were grown on soil with a $8 \mathrm{~h} \mathrm{light} / 16 \mathrm{~h}$ dark cycle, at $22{ }^{\circ} \mathrm{C}$ and $60 \%$ humidity and light intensities of 100-150 $\mu \mathrm{mol} \mathrm{m}^{-2} \mathrm{~s}^{-1}$. Ahk2,3 MIR172ox T1 plants were selected on $1 / 2$ MS agar plates $(0.22 \%(\mathrm{w} / \mathrm{v})$ MS basal salt, $0.05 \%(\mathrm{w} / \mathrm{v})$ MES, $0.5 \%(\mathrm{w} / \mathrm{v}$ ) sucrose, $0.8 \%(\mathrm{w} / \mathrm{v})$ agar, $\mathrm{pH} 5.7$ ) containing $30 \mu \mathrm{g} / \mathrm{ml}$ kanamycin and transferred to soil after 14 days. Pots of different lines were randomized by default to minimize positional effects.

Plasmid constructions for transgenic plants. For the generation of the p35S:MIR172B construct, the Gateway ${ }^{\circledast}$ system (ThermoFisher, Waltham, MA) was used. All primers used for cloning purposes are listed in Supplementary Table 4. A $35 \mathrm{~S}$ promoter fragment of the Cauliflower Mosaic Virus was PCR amplified from the vector pGWB15 $5^{90}$ using the primers p35S-F-attB4 and p35S-RattB1r, and recombined into the donor vector pDONR ${ }^{\mathrm{TM}} \mathrm{P} 4-\mathrm{P} 1 \mathrm{R}$ using Gateway ${ }^{\oplus}$ BP Clonase ${ }^{\circledast}$ Enzyme Mix (ThermoFisher, Cat. No. 11789013). MIR172B was amplified from Col-0 genomic DNA using the primers MIR172B-attB1-2_fw and MIR172B-attB2-2_rv and inserted into $\mathrm{pDONR}^{\mathrm{TM}} 221$ via $\mathrm{BP}$ reaction. The MIR172B sequence was combined with the $35 S$ promoter in $\mathrm{pK} 7 \mathrm{~m} 24 \mathrm{GW}^{91}$ to create the $p 35 S: M I R 172 B$ construct. The multisite LR reaction was performed with the Gateway ${ }^{\circledR}$ LR Clonase ${ }^{\circledR}$ II Enzyme Mix (ThermoFisher, Cat. No. 11791020). The cloned gene construct was fully sequenced to ensure that no mutation was introduced. The plasmid was transformed into the Agrobacterium tumefaciens strain GV3101 by electroporation and the resulting bacterial strain was used to transform Arabidopsis plants using the floral-dip method ${ }^{92}$.

CK induction assays. For the determination of the influence of CK on gene expression, seeds were surface-sterilized using a $1.2 \%(\mathrm{v} / \mathrm{v})$ sodium hypochlorite/ $0.01 \%(\mathrm{v} / \mathrm{v})$ Triton X-100 solution. Seedlings were grown under SD conditions for 10 days in liquid $1 / 2$ MS medium (0.22\% (w/v) MS basal salt, $0.05 \%$ (w/v) MES, $0.1 \%(\mathrm{w} / \mathrm{v})$ sucrose, $\mathrm{pH}$ adjusted to 5.7$)$. 6-Benzylaminopurine (BA) was dissolved in $1 \mathrm{M} \mathrm{KOH}$. As a control, $1 \mathrm{M} \mathrm{KOH}$ was used. Both solutions were diluted in 0.05 $(\mathrm{w} / \mathrm{v})$ MES and the $\mathrm{pH}$ was adjusted before adding them to the medium. CK application and harvesting of plant material at the different time points was conducted during the night, starting $1.5 \mathrm{~h}$ after its beginning.

RNA preparation and quantitative RT-PCR. Approximately $100 \mathrm{mg}$ of plant material was harvested and frozen in liquid nitrogen at the indicated time points. The frozen samples were ground using a Retsch mill in precooled adapters. Total RNA was extracted using TRIsure ${ }^{\mathrm{TM}}$ (Bioline) following the manufacturer's instructions. Eighty percent (v/v) ethanol was used to wash the RNA pellet, which was resuspended in $40-50 \mu \mathrm{l}$ of nuclease-free water and treated with DNase I (ThermoFisher) following the manufacturer's instructions. For normal cDNA synthesis, $1-1.5 \mu \mathrm{g}$ of total RNA was reversely transcribed using SuperScript ${ }^{\mathrm{TM}}$ III (ThermoFisher), $4.5 \mu \mathrm{M}$ of $\mathrm{N} 9$ random oligos and $2.5 \mu \mathrm{M}$ of oligo-dT 25 in a $20 \mu \mathrm{l}$ reaction. Mix 1 containing RNA, $2 \mathrm{mM}$ of dNTP mix and oligos was incubated for $5 \mathrm{~min}$ at $65^{\circ} \mathrm{C}$ and placed on ice afterwards. Mix 2 (first strand buffer, $5 \mathrm{mM}$ DTT, SuperScript ${ }^{\mathrm{TM}}$ III) was added and samples were incubated for $30 \mathrm{~min}$ at $25^{\circ} \mathrm{C}$, $60 \mathrm{~min}$ at $50^{\circ} \mathrm{C}$, and $15 \mathrm{~min}$ at $70^{\circ} \mathrm{C}$. The resulting cDNA was diluted 1:5. For detection of mature miRNAs, mix 1 contained $500 \mathrm{ng}$ of total RNA, $1 \mathrm{mM}$ of dNTP mix, $25 \mathrm{nM}$ TAFII15-StLp-cDNA_rv as an internal control for qRT analysis, and $25 \mathrm{nM}$ of the respective miRNA-specific stem-loop primer (Supplementary Table 5). Stem-loop RT primers were designed according to Chen et al. ${ }^{93}$. Following the addition of mix 2 containing first strand buffer, $4 \mathrm{mM} \mathrm{DTT,} 0.6 \mathrm{U} / \mu \mathrm{l}$ RNaseOUT (ThermoFisher), SuperScript ${ }^{\mathrm{TM}}$ III and resulting in a total volume of $12.5 \mathrm{\mu l}$, samples were incubated for $30 \mathrm{~min}$ at $16^{\circ} \mathrm{C}, 30 \mathrm{~min}$ at $50^{\circ} \mathrm{C}$, and $15 \mathrm{~min}$ at $70^{\circ} \mathrm{C}$. Undiluted cDNA was used in the qRT-PCR reactions. For qRT-PCR analyses, PROTEIN PHOSPHATASE 2A SUBUNIT A2 (PP2AA2) and TBPASSOCIATED FACTOR II 15 (TAFII15) served as reference genes. All qRT-PCR primers used in this study are listed in Supplementary Table 5. qRT-PCR was performed with the CFX96 ${ }^{\mathrm{TM}}$ Real-Time Touch System (Bio-Rad ${ }^{\oplus}$ ) using SYBR Green I as DNA-binding dye. Gene expression data analysis was carried out according to Vandesompele et al. ${ }^{94}$. For analysis of MIM156 transgene expression, the $40-\Delta \mathrm{Ct}$ method was used, as described by Morcuende et al. ${ }^{95}$.

Statistical analysis. Statistical analyses were performed using GraphPad Prism, version 8 (GraphPad Software, La Jolla, CA). Statistical tests used were all twosided and are indicated in the figure and table legends. The data was analyzed by one-way analysis of variance (ANOVA) followed by Dunnett's or Tukey's post hoc test, Kruskal-Wallis test followed by either Dunn's test or two-stage step-up procedure of Benjamini, Krieger, and Yuketieli, or two-tailed Mann-Whitney test. A pvalue or $q$-value $<0.05$ was considered to indicate a statistically significant difference. In case of transcript analyses, a 1.75-fold upregulation or downregulation compared to the respective control was chosen as threshold.
Reporting summary. Further information on research design is available in the Nature Research Reporting Summary linked to this article.

\section{Data availability}

Source data and statistical information are provided with this paper.

Received: 18 June 2021; Accepted: 1 September 2021; Published online: 05 October 2021

\section{References}

1. Teotia, S. \& Tang, G. To bloom or not to bloom: role of microRNAs in plant flowering. Mol. Plant 8, 359-377 (2015).

2. Poethig, R. S. Vegetative phase change and shoot maturation in plants. Curr. Top. Dev. Biol. 105, 125-152 (2013).

3. Srikanth, A. \& Schmid, M. Regulation of flowering time: all roads lead to Rome. Cell Mol. Life Sci. 68, 2013-2037 (2011).

4. Tsukaya, H., Shoda, K., Kim, G. T. \& Uchimiya, H. Heteroblasty in Arabidopsis thaliana (L.) Heynh. Planta 210, 536-542 (2000).

5. Usami, T., Horiguchi, G., Yano, S. \& Tsukaya, H. The more and smaller cells mutants of Arabidopsis thaliana identify novel roles for SQUAMOSA PROMOTER BINDING PROTEIN-LIKE genes in the control of heteroblasty. Development 136, 955-964 (2009).

6. Telfer, A., Bollman, K. M. \& Poethig, R. S. Phase change and the regulation of trichome distribution in Arabidopsis thaliana. Development 124, 645-654 (1997)

7. Axtell, M. J. \& Bowman, J. L. Evolution of plant microRNAs and their targets. Trends Plant Sci. 13, 343-349 (2008).

8. Cuperus, J. T., Fahlgren, N. \& Carrington, J. C. Evolution and functional diversification of MIRNA genes. Plant Cell 23, 431-442 (2011).

9. Xie, Z. et al. Expression of Arabidopsis MIRNA genes. Plant Physiol. 138, 2145-2154 (2005).

10. Breakfield, N. W. et al. High-resolution experimental and computational profiling of tissue-specific known and novel miRNAs in Arabidopsis. Genome Res. 22, 163-176 (2012).

11. Wu, G. et al. The sequential action of miR156 and miR172 regulates developmental timing in Arabidopsis. Cell 138, 750-759 (2009).

12. Wang, J. W., Czech, B. \& Weigel, D. miR156-regulated SPL transcription factors define an endogenous flowering pathway in Arabidopsis thaliana. Cell 138, 738-749 (2009).

13. He, J. et al. Threshold-dependent repression of SPL gene expression by miR156/miR157 controls vegetative phase change in Arabidopsis thaliana. PLoS Genet. 14, e1007337 (2018).

14. $\mathrm{Xu}, \mathrm{M}$. et al. Developmental functions of miR156-regulated SQUAMOSA PROMOTER BINDING PROTEIN-LIKE (SPL) genes in Arabidopsis thaliana. PLoS Genet. 12, e1006263 (2016).

15. Xing, S. et al. SPL8 and miR156-targeted $S P L$ genes redundantly regulate Arabidopsis gynoecium differential patterning. Plant J. 75, 566-577 (2013).

16. Xing, S., Salinas, M., Höhmann, S., Berndtgen, R. \& Huijser, P. MiR156targeted and nontargeted SBP-box transcription factors act in concert to secure male fertility in Arabidopsis. Plant Cell 22, 3935-3950 (2010).

17. Wu, G. \& Poethig, R. S. Temporal regulation of shoot development in Arabidopsis thaliana by miR156 and its target SPL3. Development 133 3539-3547 (2006).

18. Aukerman, M. J. \& Sakai, H. Regulation of flowering time and floral organ identity by a microRNA and its APETALA2-like target genes. Plant Cell 15, 2730-2741 (2003).

19. Chen, X. M. A microRNA as a translational repressor of APETALA2 in Arabidopsis flower development. Science 303, 2022-2025 (2004).

20. Bowman, J. L., Smyth, D. R. \& Meyerowitz, E. M. Genetic interactions among floral homeotic genes of Arabidopsis. Development 112, 1-20 (1991).

21. Jung, J. H. et al. The GIGANTEA-regulated microRNA172 mediates photoperiodic flowering independent of CONSTANS in Arabidopsis. Plant Cell 19, 2736-2748 (2007)

22. Mathieu, J., Yant, L. J., Mürdter, F., Küttner, F. \& Schmid, M. Repression of flowering by the miR172 target SMZ. PLoS Biol. 7, e1000148 (2009).

23. Huijser, P. \& Schmid, M. The control of developmental phase transitions in plants. Development 138, 4117-4129 (2011).

24. Lian, H. et al. Redundant and specific roles of individual MIR172 genes in plant development. PLoS Biol. 19, e3001044 (2021).

25. Yant, L. et al. Orchestration of the floral transition and floral development in Arabidopsis by the bifunctional transcription factor APETALA2. Plant Cell $\mathbf{2 2}$ 2156-2170 (2010).

26. Kieber, J. J. \& Schaller, G. E. Cytokinin signaling in plant development. Development 145, dev149344 (2018). 
27. Cortleven, A. et al. Cytokinin action in response to abiotic and biotic stresses in plants. Plant Cell Environ. 42, 998-1018 (2019).

28. Kieber, J. J. \& Schaller, G. E. Cytokinins. Arabidopsis Book 12, e0168 (2014).

29. Takei, K., Yamaya, T. \& Sakakibara, H. Arabidopsis CYP735A1 and CYP735A2 encode cytokinin hydroxylases that catalyze the biosynthesis of trans-zeatin. $J$. Biol. Chem. 279, 41866-41872 (2004).

30. Miyawaki, K. et al. Roles of Arabidopsis ATP/ADP isopentenyltransferases and tRNA isopentenyltransferases in cytokinin biosynthesis. Proc. Natl Acad. Sci. USA 103, 16598-16603 (2006)

31. Kakimoto, T. Identification of plant cytokinin biosynthetic enzymes as dimethylallyl diphosphate:ATP/ADP isopentenyltransferases. Plant Cell Physiol. 42, 677-685 (2001).

32. Takei, K., Sakakibara, H. \& Sugiyama, T. Identification of genes encoding adenylate isopentenyltransferase, a cytokinin biosynthesis enzyme, in Arabidopsis thaliana. J. Biol. Chem. 276, 26405-26410 (2001).

33. Kuroha, T. et al. Functional analyses of LONELY GUY cytokinin-activating enzymes reveal the importance of the direct activation pathway in Arabidopsis. Plant Cell 21, 3152-3169 (2009).

34. Kurakawa, T. et al. Direct control of shoot meristem activity by a cytokininactivating enzyme. Nature 445, 652-655 (2007).

35. Werner, T., Köllmer, I., Bartrina, I., Holst, K. \& Schmülling, T. New insights into the biology of cytokinin degradation. Plant Biol. 8, 371-381 (2006).

36. Hou, B., Lim, E. K., Higgins, G. S. \& Bowles, D. J. N-glucosylation of cytokinins by glycosyltransferases of Arabidopsis thaliana. J. Biol. Chem. 279, 47822-47832 (2004).

37. Inoue, $\mathrm{T}$. et al. Identification of CRE1 as a cytokinin receptor from Arabidopsis. Nature 409, 1060-1063 (2001).

38. Yamada, H. et al. The Arabidopsis AHK4 histidine kinase is a cytokininbinding receptor that transduces cytokinin signals across the membrane. Plant Cell Physiol. 42, 1017-1023 (2001).

39. Ueguchi, C., Koizumi, H., Suzuki, T. \& Mizuno, T. Novel family of sensor histidine kinase genes in Arabidopsis thaliana. Plant Cell Physiol. 42, 231-235 (2001).

40. Suzuki, T. et al. The Arabidopsis sensor His-kinase, AHK4, can respond to cytokinins. Plant Cell Physiol. 42, 107-113 (2001).

41. Hwang, I. \& Sheen, J. Two-component circuitry in Arabidopsis cytokinin signal transduction. Nature 413, 383-389 (2001).

42. Brenner, W. G., Ramireddy, E., Heyl, A. \& Schmülling, T. Gene regulation by cytokinin in Arabidopsis. Front. Plant Sci. 3, 8 (2012).

43. Galvão, V. C., Horrer, D., Kuttner, F. \& Schmid, M. Spatial control of flowering by DELLA proteins in Arabidopsis thaliana. Development 139, 4072-4082 (2012)

44. Yu, S. et al. Gibberellin regulates the Arabidopsis floral transition through miR156-targeted SQUAMOSA PROMOTER BINDING-LIKE transcription factors. Plant Cell 24, 3320-3332 (2012).

45. Jung, J. H., Ju, Y., Seo, P. J., Lee, J. H. \& Park, C. M. The SOC1-SPL module integrates photoperiod and gibberellic acid signals to control flowering time in Arabidopsis. Plant J. 69, 577-588 (2012).

46. Chien, J. C. \& Sussex, I. M. Differential regulation of trichome formation on the adaxial and abaxial leaf surfaces by gibberellins and photoperiod in Arabidopsis thaliana (L.) Heynh. Plant Physiol. 111, 1321-1328 (1996).

47. Dill, A. \& Sun, T. Synergistic derepression of gibberellin signaling by removing RGA and GAI function in Arabidopsis thaliana. Genetics 159, 777-785 (2001).

48. Bartrina, I. et al. Gain-of-function mutants of the cytokinin receptors AHK2 and AHK3 regulate plant organ size, flowering time and plant longevity. Plant Physiol. 173, 1783-1797 (2017).

49. Chickarmane, V. S., Gordon, S. P., Tarr, P. T., Heisler, M. G. \& Meyerowitz, E. M. Cytokinin signaling as a positional cue for patterning the apical-basal axis of the growing Arabidopsis shoot meristem. Proc. Natl Acad. Sci. USA 109, 4002-4007 (2012).

50. Gruel, J. et al. An epidermis-driven mechanism positions and scales stem cell niches in plants. Sci. Adv. 2, e1500989 (2016).

51. Bartrina, I., Otto, E., Strnad, M., Werner, T. \& Schmülling, T. Cytokinin regulates the activity of reproductive meristems, flower organ size, ovule formation, and thus seed yield in Arabidopsis thaliana. Plant Cell 23, 69-80 (2011).

52. Mason, M. G., Li, J., Mathews, D. E., Kieber, J. J. \& Schaller, G. E. Type-B response regulators display overlapping expression patterns in. Arabidopsis. Plant Physiol. 135, 927-937 (2004)

53. Tajima, Y. et al. Comparative studies on the type-B response regulators revealing their distinctive properties in the His-to-Asp phosphorelay signal transduction of Arabidopsis thaliana. Plant Cell Physiol. 45, 28-39 (2004).

54. Ishida, K., Yamashino, T., Yokoyama, A. \& Mizuno, T. Three type-B response regulators, ARR1, ARR10 and ARR12, play essential but redundant roles in cytokinin signal transduction throughout the life cycle of Arabidopsis thaliana. Plant Cell Physiol. 49, 47-57 (2008).

55. Argyros, R. D. et al. Type B response regulators of Arabidopsis play key roles in cytokinin signaling and plant development. Plant Cell 20, 2102-2116 (2008).
56. Franco-Zorrilla, J. M. et al. Target mimicry provides a new mechanism for regulation of microRNA activity. Nat. Genet. 39, 1033-1037 (2007).

57. Todesco, M., Rubio-Somoza, I., Paz-Ares, J. \& Weigel, D. A collection of target mimics for comprehensive analysis of microRNA function in Arabidopsis thaliana. PLoS Genet. 6, el001031 (2010).

58. Schwarz, S., Grande, A. V., Bujdoso, N., Saedler, H. \& Huijser, P. The microRNA regulated SBP-box genes SPL9 and SPL15 control shoot maturation in Arabidopsis. Plant Mol. Biol. 67, 183-195 (2008).

59. Schwab, R. et al. Specific effects of microRNAs on the plant transcriptome. Dev. Cell 8, 517-527 (2005)

60. Riefler, M., Novak, O., Strnad, M. \& Schmülling, T. Arabidopsis cytokinin receptor mutants reveal functions in shoot growth, leaf senescence, seed size, germination, root development, and cytokinin metabolism. Plant Cell 18, 40-54 (2006).

61. Lauter, N., Kampani, A., Carlson, S., Goebel, M. \& Moose, S. P. microRNA172 down-regulates glossy15 to promote vegetative phase change in maize. Proc. Natl Acad. Sci. USA 102, 9412-9417 (2005).

62. Schäfer, M. et al. The role of cis-zeatin-type cytokinins in plant growth regulation and mediating responses to environmental interactions. J. Exp. Bot. 66, 4873-4884 (2015)

63. Ko, D. et al. Arabidopsis ABCG14 is essential for the root-to-shoot translocation of cytokinin. Proc. Natl Acad. Sci. USA 111, 7150-7155 (2014).

64. Zhang, K. et al. Arabidopsis ABCG14 protein controls the acropetal translocation of root-synthesized cytokinins. Nat. Commun. 5, 3274 (2014).

65. Kiba, T., Takei, K., Kojima, M. \& Sakakibara, H. Side-chain modification of cytokinins controls shoot growth in Arabidopsis. Dev. Cell 27, 452-461 (2013).

66. Yang, L., Conway, S. R. \& Poethig, R. S. Vegetative phase change is mediated by a leaf-derived signal that represses the transcription of miR156. Development 138, 245-249 (2011)

67. Fouracre, J. P. \& Poethig, R. S. Role for the shoot apical meristem in the specification of juvenile leaf identity in Arabidopsis. Proc. Natl Acad. Sci. USA 116, 10168-10177 (2019).

68. Bernier, G. My favourite flowering image: the role of cytokinin as a flowering signal. J. Exp. Bot. 64, 5795-5799 (2013).

69. D'Aloia, M. et al. Cytokinin promotes flowering of Arabidopsis via transcriptional activation of the FT paralogue TSF. Plant J. 65, 972-979 (2011).

70. Romanov, G. A., Lomin, S. N. \& Schmülling, T. Biochemical characteristics and ligand-binding properties of Arabidopsis cytokinin receptor AHK3 compared to CRE1/AHK4 as revealed by a direct binding assay. J. Exp. Bot. 57, 4051-4058 (2006).

71. Stolz, A. et al. The specificity of cytokinin signalling in Arabidopsis thaliana is mediated by differing ligand affinities and expression profiles of the receptors. Plant J. 67, 157-168 (2011).

72. Mason, $M$. G. et al. Multiple type-B response regulators mediate cytokinin signal transduction in Arabidopsis. Plant Cell 17, 3007-3018 (2005).

73. Choi, J. et al. The cytokinin-activated transcription factor ARR2 promotes plant immunity via TGA3/NPR1-dependent salicylic acid signaling in Arabidopsis. Dev. Cell 19, 284-295 (2010).

74. Nitschke, S. et al. Circadian stress regimes affect the circadian clock and cause jasmonic acid-dependent cell death in cytokinin-deficient Arabidopsis plants. Plant Cell 28, 1616-1639 (2016)

75. Frank, M., Cortleven, A., Novák, O. \& Schmülling, T. Root-derived transzeatin cytokinin protects Arabidopsis plants against photoperiod stress. Plant Cell Environ. 43, 2637-2649 (2020).

76. Kim, H. J. et al. Cytokinin-mediated control of leaf longevity by AHK3 through phosphorylation of ARR2 in Arabidopsis. Proc. Natl Acad. Sci. USA 103, 814-819 (2006).

77. Rhoades, M. W. et al. Prediction of plant microRNA targets. Cell 110, 513-520 (2002).

78. Cardon, G. et al. Molecular characterisation of the Arabidopsis SBP-box genes Gene 237, 91-104 (1999).

79. Zubo, Y. O. et al. Cytokinin induces genome-wide binding of the type-B response regulator ARR10 to regulate growth and development in Arabidopsis. Proc. Natl Acad. Sci. USA 114, E5995-E6004 (2017)

80. Xie, M. et al. A B-ARR-mediated cytokinin transcriptional network directs hormone cross-regulation and shoot development. Nat. Commun. 9, 1604 (2018).

81. Hyun, Y. et al. Multi-layered regulation of SPL15 and cooperation with SOC1 integrate endogenous flowering pathways at the Arabidopsis shoot meristem. Dev. Cell 37, 254-266 (2016).

82. Zhang, T. Q. et al. An intrinsic microRNA timer regulates progressive decline in shoot regenerative capacity in plants. Plant Cell 27, 349-360 (2015)

83. Jung, J. H., Lee, S., Yun, J., Lee, M. \& Park, C. M. The miR172 target TOE3 represses AGAMOUS expression during Arabidopsis floral patterning. Plant Sci. 215-216, 29-38 (2014).

84. Higuchi, M. et al. In planta functions of the Arabidopsis cytokinin receptor family. Proc. Natl Acad. Sci. USA 101, 8821-8826 (2004). 
85. Yang, L., Xu, M., Koo, Y., He, J. \& Poethig, R. S. Sugar promotes vegetative phase change in Arabidopsis thaliana by repressing the expression of MIR156A and MIR156C. Elife 2, e00260 (2013).

86. $\mathrm{Yu}, \mathrm{S}$. et al. Sugar is an endogenous cue for juvenile-to-adult phase transition in plants. Elife 2, e00269 (2013).

87. Ponnu, J. et al. The trehalose 6-phosphate pathway impacts vegetative phase change in Arabidopsis thaliana. Plant J. 104, 768-780 (2020).

88. Lawrence, E. H., Springer, C. J., Helliker, B. R. \& Poethig, R. S. MicroRNA156mediated changes in leaf composition lead to altered photosynthetic traits during vegetative phase change. New Phytol. 231, 1008-1022 (2020).

89. Wang, L. et al. A spatiotemporally regulated transcriptional complex underlies heteroblastic development of leaf hairs in Arabidopsis thaliana. EMBO J. 38, e100063 (2019).

90. Nakagawa, T. et al. Development of series of gateway binary vectors, pGWBs, for realizing efficient construction of fusion genes for plant transformation. $J$. Biosci. Bioeng. 104, 34-41 (2007).

91. Karimi, M., De Meyer, B. \& Hilson, P. Modular cloning in plant cells. Trends Plant Sci. 10, 103-105 (2005).

92. Clough, S. J. \& Bent, A. F. Floral dip: a simplified method for Agrobacteriummediated transformation of Arabidopsis thaliana. Plant J. 16, 735-743 (1998).

93. Chen, C. et al. Real-time quantification of microRNAs by stem-loop RT-PCR. Nucleic Acids Res. 33, e179 (2005).

94. Vandesompele, J. et al. Accurate normalization of real-time quantitative RTPCR data by geometric averaging of multiple internal control genes. Genome Biol. 3, 0031-0034.0011 (2002). research0034.

95. Morcuende, R. et al. Genome-wide reprogramming of metabolism and regulatory networks of Arabidopsis in response to phosphorus. Plant Cell Environ. 30, 85-112 (2007).

\section{Acknowledgements}

We acknowledge excellent technical assistance by Gabriele Grüschow and thank Tim Krahn and Jutta Hoffmann for their contributions made during their bachelor thesis. We thank Youngsook Lee and the Nottingham Arabidopsis Stock Centre for providing seeds. The vector $\mathrm{pDONR}{ }^{\mathrm{TM}} \mathrm{P} 4-\mathrm{P} 1 \mathrm{R} / \mathrm{p} 35 \mathrm{~S}$ was kindly provided by Elisabeth Otto.

\section{Author contributions}

S.W. discovered the influence of cytokinin on vegetative phase change; S.W., I.B., and T.S. developed the project; S.W. performed experiments; S.W., I.B., and T.S. analyzed data; S.W. and T.S. wrote the article, I.B. read and contributed to previous versions and approved the final version.

\section{Funding}

Open Access funding enabled and organized by Projekt DEAL.

\section{Competing interests}

The authors declare no competing interests.

\section{Additional information}

Supplementary information The online version contains supplementary material available at https://doi.org/10.1038/s41467-021-26088-z.

Correspondence and requests for materials should be addressed to Thomas Schmülling

Peer review information Nature Communications thanks Jia-Wei Wang and the other, anonymous, reviewer(s) for their contribution to the peer review of this work. Peer reviewer reports are available.

Reprints and permission information is available at http://www.nature.com/reprints

Publisher's note Springer Nature remains neutral with regard to jurisdictional claims in published maps and institutional affiliations.

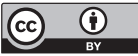

Open Access This article is licensed under a Creative Commons Attribution 4.0 International License, which permits use, sharing, adaptation, distribution and reproduction in any medium or format, as long as you give appropriate credit to the original author(s) and the source, provide a link to the Creative Commons license, and indicate if changes were made. The images or other third party material in this article are included in the article's Creative Commons license, unless indicated otherwise in a credit line to the material. If material is not included in the article's Creative Commons license and your intended use is not permitted by statutory regulation or exceeds the permitted use, you will need to obtain permission directly from the copyright holder. To view a copy of this license, visit http://creativecommons.org/ licenses/by/4.0/.

(C) The Author(s) 2021 